\title{
II)
}

\section{A Generic Approach for High-throughput Blood Analysis}

\section{Sophie Bravo-Veyrat and Gérard Hopfgartner*}

${ }^{*}$ Correspondence: Prof. Dr. G. Hopfgartner, University of Geneva, Life Sciences Mass Spectrometry, Department of Inorganic and Analytical Chemistry,

24 Quai Ernest-Ansermet, CH-1211 Genève 4, E-mail: gerard.hopfgartner@unige.ch

Keywords: Blood - Differential mobility spectrometry · MRM . Quantification · Trap/Elute LC

During the 42 days of legal storage of blood bags, red blood cells can be altered due to an increase of oxidative stress degrading blood quality prior to transfusion. Oxidative stress can be measured by the ratio between the accurate concentrations of reduced and oxidized glutathione (respectively GSH and GSSG).

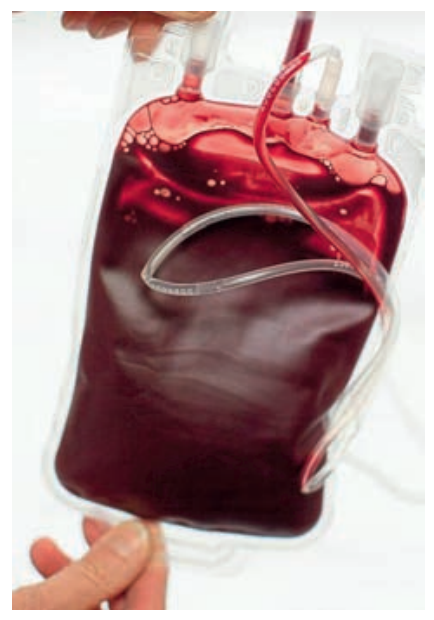

Donated blood is precious and should be of highest possible quality.

Quantitative liquid chromatography coupled to multiple reaction monitoring mass spectrometry (LC-MRM/MS) is the current gold standard for quantitative bioanalysis, and different assays have been reported for the quantification of GSH and GSSG. However, analysis times of 5 to 10 minutes limit their application for high-throughput analysis. Flow-injection analysis in MRM is not an alternative as matrix effects during ionization alter the limit of quantification, and non-separated endogenous analytes affect the selectivity of the assay. A decrease of analytical time while maintaining good precision and accuracy passes by the shortening of the column length. Replacing a $50 \mathrm{~mm}$ Hypercarb column for a $10 \mathrm{~mm} \mathrm{C18}$ column (short LC) gives a 1-minute runtime but inaccurate and non-precise quantitative results. As blood cannot be injected directly into an LC column a protein precipitation (PP) step is mandatory. The use of organic solvent would require an additional evaporation/reconstitution step. Perchloric acid is an efficient PP agent and allows to inject a large volume onto a trapping column. In the present work a short LC column was used in trap/elute mode, with an in-line front flush preconcentration performed with ion-pairing trifluoroacetic acid. MS sensitivity was improved by backflush elution with formic acid. One-dimensional separation with the short LC column allowed to control suppression effects but selectivity was still lacking for GSH and GSSG. Modifier-assisted differential mobility spectrometry (DMS) was added as a second dimension of separation without compromising analysis time. This 'in-space' separation of GSH and GSSG, based on their compensation voltage $(\mathrm{CoV})$ in the DMS cell, was tuned with the addition of modifiers. Thereby, the best resolution was obtained with ethanol, allowing to separate GSH and GSSG with different CoV values. With this 2D separation, oxidative stress could be measured in 10 human donors in 1.5 min per sample with accuracy and precision.

This generic approach combining perchloric protein precipitation, multidimensional trap/elute short $\mathrm{LC}$ column, and differential mobility spectrometry with MS detection is an alternative to LC for high-throughput bioanalysis with the capability to quantify 400 samples in 10 hours.

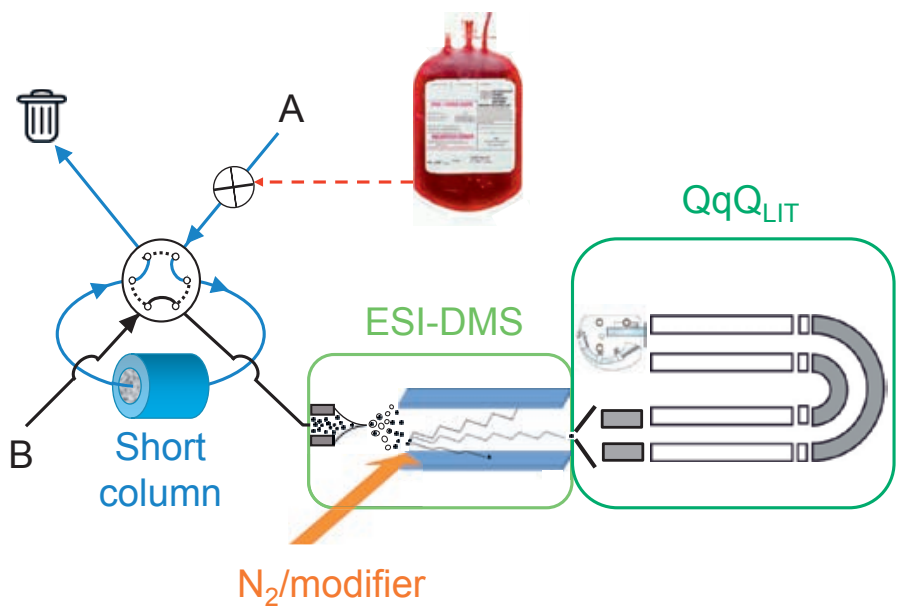

Generic bioanalytical workflow of whole blood sample combining short LC column-switching and differential mobility spectrometry assisted by modifier, with a $\mathrm{QqQ}_{\mathrm{LIT}}$ mass spectrometer.

\section{References}

D. Giustarini, D. Tsikas, G. Colombo, A. Milzani, I. Dalle-Donne, P. Fanti, R. Rossi, J. Chromatogr. B 2016, 1019, 21.

G. Hopfgartner, E. Bourgogne, Mass Spectrom. Rev. 2003, 22, 195.

S. Bravo-Veyrat, G. Hopfgartner, Anal. Bioanal. Chem. 2018, 410, 7153. 